\title{
The microscopic origin of DMI in magnetic bilayers and prediction of giant DMI in new bilayers
}

\author{
Priyamvada Jadaun $\mathbb{D}^{1 凶}$, Leonard F. Register ${ }^{1}$ and Sanjay K. Banerjee ${ }^{1}$
}

Skyrmions are widely regarded as promising candidates for emergent spintronic devices. Dzyaloshinskii-Moriya interaction (DMI) is often critical to the generation and manipulation of skyrmions. However, there is a fundamental lack of understanding of the origin of DMI or the mechanism by which DMI generates skyrmions in magnetic bilayers. Very little is known of the material parameters that determine the value of DMI. This knowledge is vital for rational design of skyrmion materials and further development of skyrmion technology. To address this important problem, we investigate DMI in magnetic bilayers using first principles. We present a new theoretical model that explains the microscopic origin of DMI in magnetic bilayers. We demonstrate that DMI depends on two parameters, interfacial hybridization and orbital contributions of the heavy metal. Using these parameters, we explain the trend of DMI observed. We also report four new materials systems with giant DMI and new designs for magnetic multilayers that are expected to outperform the best materials known so far. Our results present a notably new understanding of DMI, uncover highly promising materials and put forth pathways for the controlled generation of skyrmions.

npj Computational Materials (2020)6:88; https://doi.org/10.1038/s41524-020-00351-1

\section{INTRODUCTION}

As our society's need to store data continuously increases, we will soon reach the performance limits of current memory devices ${ }^{1}$. Magnetic skyrmions are widely believed to be one of the most promising candidates for next-generation memory technology. A magnetic skyrmion is a local whirl of spins in a magnetic material with a fixed chirality ${ }^{2,3}$. It is topologically protected against deformation into other magnetic states, and against disorder and perturbation. The stability of skyrmions, their small size and their responsiveness to tiny electrical current densities $\left(\sim 10^{6} \mathrm{~A} / \mathrm{m}^{2}\right)$, make these skyrmions ideal for enabling ultra-dense, low-energy memory devices ${ }^{3}$. One of the important mechanisms controlling the generation and stability of skyrmions and other magnetic topological defects ${ }^{4}$ is the Dzyaloshinskii-Moriya interaction $(\mathrm{DMI})^{5,6}$.

Despite its fundamental importance, the microscopic origin of $\mathrm{DMI}$ in magnetic bilayers is not well understood. Originally derived for magnetic insulators, the theory developed by Moriya ${ }^{6}$ does not help us understand the trend of DMI observed in magnetic bilayers. Recent works have tried to uncover the microscopic origin of DMI in magnetic bilayers. One model proposes that the source of DMI in bilayers is the proximity-induced magnetic moments in the heavy metal(HM) layers ${ }^{7}$. However, this proposal is contradicted by another report ${ }^{8}$. Other interesting studies point out that HM-FM hybridization is a primary factor in controlling the strength of $\mathrm{DMI}^{9,10}$, with which our model agrees. However, the proposition of $^{9}$ that the sign of DMI follows FM 3d band filling is contradicted by the large, negative DMI seen in $\mathrm{Ir} / \mathrm{Fe}^{11,12}$ and both positive and negative signs of $\mathrm{DMl}$ reported for $\mathrm{HM} / \mathrm{Co}^{8,13}$. Similarly, the proposal that DMI is controlled solely by the spinpolarized $\mathrm{HM}-5 \mathrm{~d}$ states in $\mathrm{Pt} / \mathrm{FM}^{10}$ is contradicted by experimental reports of opposite signs of $\mathrm{DMl}$ for $\mathrm{Pt} / \mathrm{Co}^{14}$ and $\mathrm{Pt} / \mathrm{Ni}^{15}$.

There is thus a gap in our understanding of the relationship of DMI with electronic structure, and of the mechanism by which DMI produces magnetic textures. This gap hampers our ability to design materials for enhanced DMl, or to control the strength or sign of DMI, which can help the development of controllable skyrmion technology ${ }^{16}$. In this paper, we address this gap and present a model to explain the microscopic origin of DMI in metallic bilayers. We calculate the DMI values in a series of heavy metal/ferromagnet (HM/FM) bilayers and explain the trend of DMI observed. In particular, we show that the value of DMI depends on two factors, namely, HM-FM hybridization and HM spin mixing terms. The latter are determined by the contribution of specific $\mathrm{HM}$ orbitals to the HM/FM bandstructure. We emphasize that the $\mathrm{HM} / \mathrm{FM}$ bandstructure, and consequently the DMl sign, depends on the choice of both the FM as well as the HM. We also derive a theory for the mechanism by which spin orbit coupling (SOC) generates spin textures. Our results present a significantly new understanding of DMI. They also unveil important avenues to control the strength and sign of DMl, thereby advancing the controlled generation or annihilation of skyrmions.

Real world applications in emergent memory technology would ideally require skyrmions of size $\leq 10 \mathrm{~nm}$ that are stable at room temperature ${ }^{2}$. Ultrathin HM/FM films have been shown to host small skyrmions $(\sim 3-8 \mathrm{~nm})$ stabilized by DMI, but so far they exist only at low temperatures $(<30 \mathrm{~K})^{12,17,18}$. On the other hand, HM/ FM multilayer stacks can host skyrmions at room temperature, but so far they have been larger than $30 \mathrm{~nm}$ in diameter ${ }^{1,19-22}$. Recently, it has been proposed that frustrated magnets can also host skyrmions that are stabilized by competing ferromagnetic nearest-neighbor and antiferromagnetic next-nearest-neighbor interactions ${ }^{23-26}$. The existence of such skyrmions has been very recently shown for $\mathrm{Gd}_{2} \mathrm{PdSi}_{3}{ }^{27}$. While these skyrmions are very small in size (approximately a few nms), so far, they have only been observed at low temperatures $(<20 \mathrm{~K})$ as well.

In HM/FM bilayers, the size and stability of DMl-stabilizedskyrmions is decided by the interplay between multiple variables, including, the DMI, the exchange constant, the out-of plane anisotropy and dipolar fields ${ }^{28-31}$. Therefore, to realize skyrmion based emergent memories, it will be important to identify materials with the appropriate combinations of these parameters.

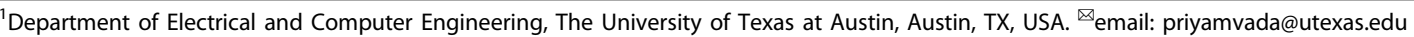


As a general rule, a large ratio of DMl to exchange constant encourages a quicker rotation of the spin, and reduces the size of the skyrmion (in the absence of interactions like edge effects) ${ }^{3}$. Thus, materials with enhanced values of DMI can host skyrmions that are stable at higher temperatures and small in size. Here, we report new bilayers that demonstrate giant $\mathrm{DMI}$, up to twice the largest value known so far. We also present new material designs for magnetic multilayers that show enhanced DMI. We note that starting with an HM-FM bilayer with a giant interfacial DMI, fine tuning of DMI and exchange values is possible by further engineering of the stacks ${ }^{32,33}$. Large DMI also enables the formation of other chiral spin structures, like chiral domain walls, which are relevant to next-generation data storage devices ${ }^{34}$. Our results thus significantly advance the field by presenting new materials, which show promise for enabling skyrmion device technology.

\section{RESULTS}

New materials with giant DMI

Our results for DMI are shown in Fig. 1. Our values show excellent agreement with literature, except for $\mathrm{Re} / \mathrm{Fe}$, where our DMI is roughly half that predicted by Simon et al. ${ }^{35}$. A significant result is the giant DMI seen in six bilayers, namely, Re/Fe, Os/Fe, Re/Co, Os/ $\mathrm{Co}, \mathrm{Os} / \mathrm{Ni}$ and hexagonal Bismuth(hBi)/Ni. These materials show a $\mathrm{DMI}(d)$ up to twice the largest currently known values of $1.5 \mathrm{meV} /$ atom for $\mathrm{Co} / \mathrm{Pt}^{8},-1.9 \mathrm{meV} / \mathrm{atom}$ for $\mathrm{Ir} / \mathrm{Fe}^{8}$ and $-1.04 \mathrm{meV} /$ atom for $\mathrm{Ir} / \mathrm{Co}^{36}$. Note our predicted values of $\mathrm{DMI}(d)$ for these materials are $2.6 \mathrm{meV} /$ atom for $\mathrm{Co} / \mathrm{Pt},-1.8 \mathrm{meV} /$ atom for $\mathrm{Ir} / \mathrm{Fe}$ and -1.8 $\mathrm{meV} /$ atom for Ir/Co. Predictions of large DMI in Re/Co, Os/Co, Os/ $\mathrm{Ni}$ and $\mathrm{hBi} / \mathrm{Ni}$ have not been reported before. As a possible quantum spin Hall material ${ }^{37}$, hexagonal $\mathrm{Bi}$ is particularly noteworthy, as it can help drive skyrmions via a large spin Hall current. A useful materials design strategy for enhancing DMI has been the addition of DMI of opposite sign from successive interfaces ${ }^{38,39}$, as implemented in $\mathrm{Pt} / \mathrm{Co} / \mathrm{Fe} / \mathrm{Ir}$ multilayers. Our results make it possible to achieve even larger additive DMls in simpler multilayer structures, such as $\mathrm{Pt} / \mathrm{Co} / \mathrm{Os}$ and $\mathrm{Pt} / \mathrm{Co} / \mathrm{Re}$.

Plot of DMI with Heavy Metal
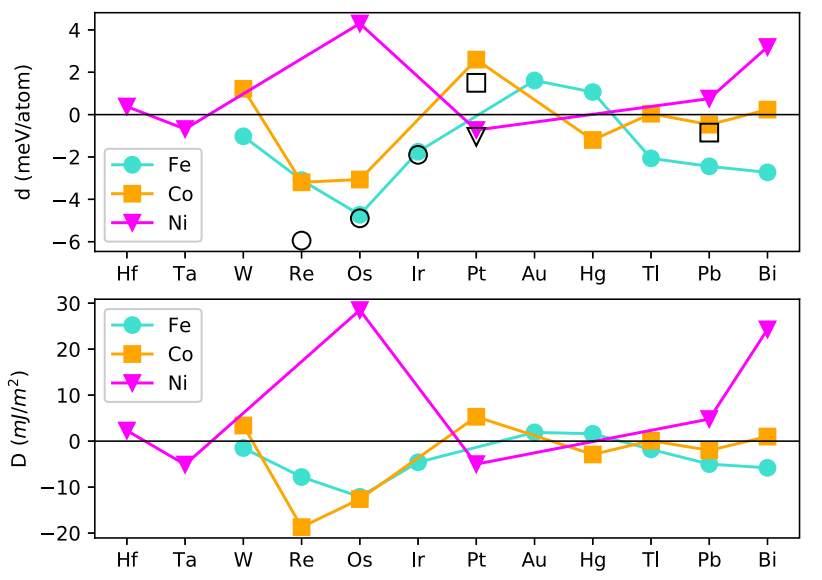

Fig. 1 Plots of Dzyaloshinskii-Moriya interaction (DMI) for a series of HM/FM bilayers (HM: Hf-Bi). Our DMI results are plotted with filled markers, whereas corresponding DMI values in literature are plotted using unfilled black markers. Circles represent $\mathrm{Fe}$, squares represent $\mathrm{Co}$ and triangles represent $\mathrm{Ni}$. Reference values are taken from ref. ${ }^{35}$ for $\mathrm{Re} / \mathrm{Fe}$, Os/Fe Ir/Fe; from ref. ${ }^{36} \mathrm{for} \mathrm{Ir} / \mathrm{Co}$; from ref. ${ }^{8}$ for $\mathrm{Pt} / \mathrm{Co}$; from ref. ${ }^{11}$ for $\mathrm{Pb} / \mathrm{Co}$; and from ref. ${ }^{15}$ for $\mathrm{Pt} / \mathrm{Ni}$. The unit of $D$ is $m J /(m-B M)^{2}$, where $B M$ is the Bohr magneton.

\section{DISCUSSION}

We now present the analysis of our results and develop a model for the microscopic origin of DMI. We demonstrate that DMI depends on two parameters, HM-FM hybridization and the presence of specific HM orbitals in the bandstructure. We find that the strength of DMI is controlled by the relative alignment of HM-FM bands. Here we show that the sign of DMI is controlled by orbital contributions from the HM rather than the $3 \mathrm{~d}$ orbital filling of the FM. Our work thus notably alters the current understanding of DMI in magnetic bilayers.

We now show that the strength of DMI $(d)$ is principally determined by the HM-FM hybridization. To demonstrate this, we examine the projected density of states (p-DOS), plotted in Figs. 2, 3, and relative band alignments between the HM and FM layers. We define band overlap (BO) as the amount of overlap exhibited by the FM-3d bands with the HM- $5 d$ bands ( $6 p$ bands) for the $5 d(6 p)$ series of HMs. We expect that the greater the $\mathrm{BO}$, the greater the HM-FM hybridization. Upon visual inspection, we clearly see that $d$ strongly depends on BO. In particular, a large BO is a necessary but not sufficient condition to obtaining large $d$. Since $\mathrm{Fe}, \mathrm{Co}$ and $\mathrm{Ni}$ have $\mathrm{d} 6-\mathrm{d} 8$ electron configurations, the $3 \mathrm{~d}-5 \mathrm{~d}$ band overlap is maximized for $\mathrm{Re}, \mathrm{Os}$, Ir and $\mathrm{Pt}(\mathrm{d} 5-\mathrm{d} 8$ ), giving rise to maximum DMI strength for these materials. Similarly for HM-6p elements, $d$ increases for increasing BO. There are notable outliers, namely, Ir/ $\mathrm{Fe}, \mathrm{Pt} / \mathrm{Co}$ and $\mathrm{Pt} / \mathrm{Ni}$ (and $\mathrm{hBi} / \mathrm{Co}$ for $\mathrm{HM}-6 \mathrm{p}$ ) where the $\mathrm{DMl}$ obtained is smaller than what would be expected from $\mathrm{BO}$ alone. We explain the reason for these exceptions later.

For the $\mathrm{HM}-5 \mathrm{~d} /$ Fe series, we observe a trend of $\left|d_{\beta W / F e}\right|<\mid d_{A u}$ $\mathrm{Fe}|<| d_{\text {Re/Fe }}|<| d_{\text {OS/Fe }} \mid$. This is easily explained as the BO also increases in this order, as shown in Fig. 2. Similarly, for the HM-5d/ Co series, we note a trend of $\left|d_{\mathrm{Hg} / \mathrm{Co}}\right| \sim\left|d_{a W / \mathrm{CO}}\right|<\left|d_{P t / \mathrm{Co}}\right|<\left|d_{\mathrm{OS} / \mathrm{Co}}\right| \sim$ $\left|d_{R e / C o}\right|$. This is explained by the large $\mathrm{BO}$ seen for $\mathrm{Pt} / \mathrm{Co}, \mathrm{Os} / \mathrm{Co}$ and $\mathrm{Re} / \mathrm{Co}$, as shown in Fig. 3. The HM-Ni series also follows similar trends with increasing $d$ for increasing $\mathrm{BO}$. As noted before, $\mathrm{Ir} / \mathrm{Fe}$, $\mathrm{Pt} / \mathrm{Co}$ and $\mathrm{Pt} / \mathrm{Ni}$ have a smaller $d$ than one would expect simply from BO. The only other outlier is $\mathrm{Hg}$, which both for $\mathrm{Fe}$ and $\mathrm{Co}$ has a DMI which is small, but larger than expected from simply $3 d-5 d \mathrm{BO} . \mathrm{Hg} / \mathrm{FM}$ results suggest that there might be other, smaller contributions to HM/FM hybridization in addition to the $3 \mathrm{~d}-5 \mathrm{~d}$ BO. For the HM-6p/FM series, the observed trend of $\left|d_{T / / F e}\right|$ $\lesssim\left|d_{P b / F e}\right| \lesssim\left|d_{B i / F e}\right|$ and $\left|d_{T I / C o}\right|<\left|d_{B i / C o}\right|<\left|d_{P b / C o}\right|$ both correlate well with the increasing order of BO. Overall, our results show that HM-FM band overlap has a significant control over the strength of DMI. We explain the cause of this relationship later.

The sign of DMI is important as it decides the sense of rotation of a magnetic texture. We note that DMI's sign does not follow FM $3 \mathrm{~d}$ band filling and can in fact change even for the same FM. Here we investigate the origin of this sign. We derive a theory for the mechanism by which SOC generates magnetic textures, and demonstrate that the sign of DMl is controlled by the relative presence of $\mathrm{HM}$ orbitals in the bandstructure. Our findings bring to light the source of the DMl's sign and point to new pathways for controlling skyrmion chirality, with significant impact to future technology.

We use first order perturbation theory to derive the mechanism by which SOC leads to the creation of magnetic textures. We build upon an important previous work on the Berry phase theory of $\mathrm{DMI}^{40,41}$. According to this Berry phase theory, the rotation of the magnetic moment of an electron alters the free energy, via exchange interaction, and gives rise to DMI. Here we demonstrate how SOC leads to the rotation of magnetic moment in the first place, which subsequently creates magnetic textures and generates DMI. Our derivation applies specifically to the hedgehog-like (Néel) skyrmions found in magnetic multilayers ${ }^{42}$, as shown in Fig. 4.

Consider a HM/FM bilayer system. In the absence of SOC, the FM has a constant magnetization. Let $\psi_{\vec{k} n}^{0}$ represent the 

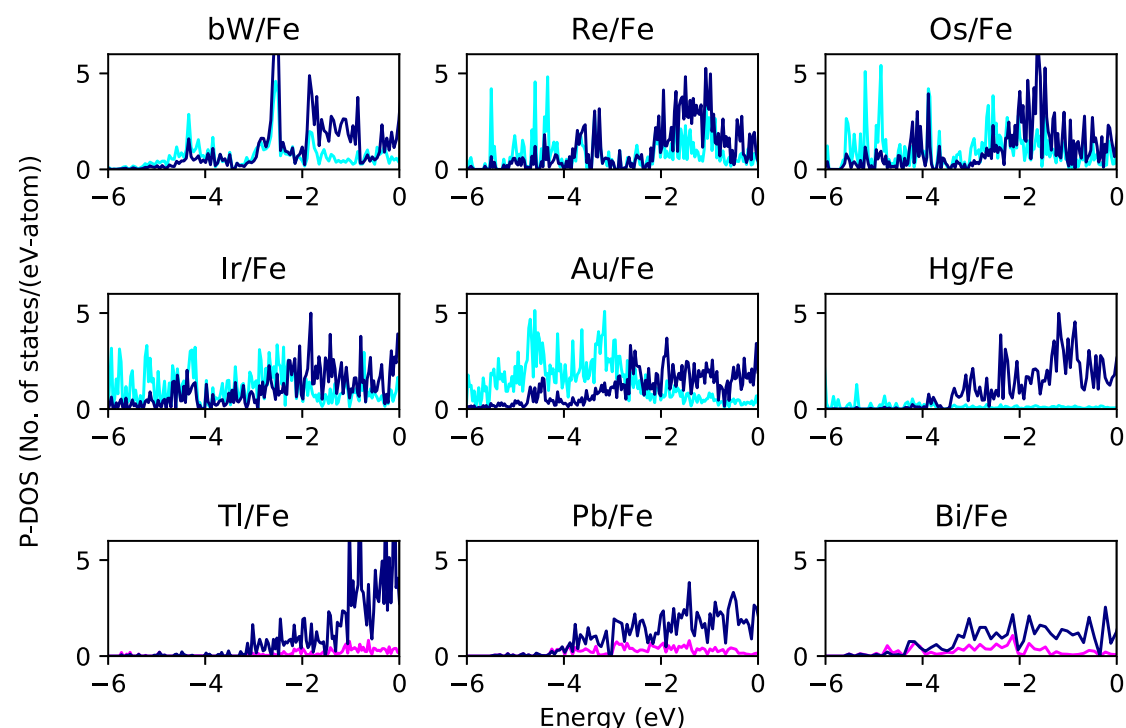

Fig. 2 Plots of projected density of states (p-DOS) and band alignment for HM/Fe bilayers. P-DOS for Fe-3d is plotted in navy blue while $\mathrm{p}$-DOS for HM-5d is plotted in cyan and that for HM-6p is plotted in magenta. Fermi energy is set at $0 \mathrm{eV}$.
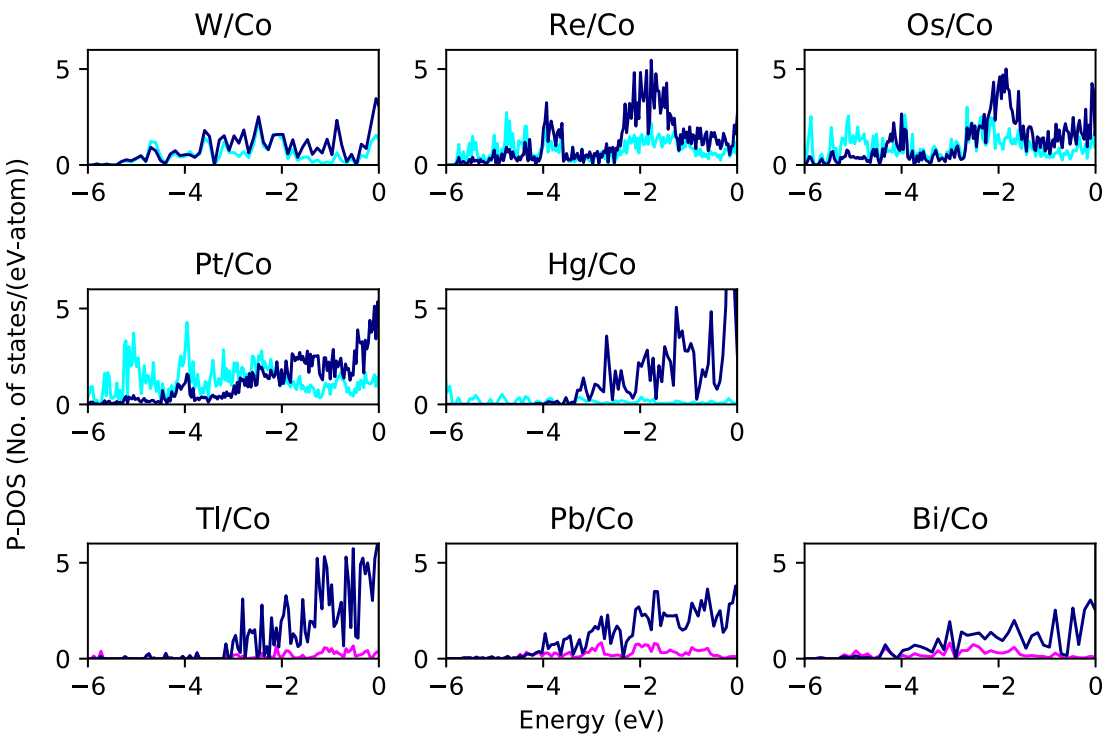

Fig. 3 Plots of projected density of states (p-DOS) and band alignment for HM/Co bilayers. P-DOS for Co-3d is plotted in navy blue while $\mathrm{p}$-DOS for HM-5d is plotted in cyan and that for HM-6p is plotted in magenta. Fermi energy is set at $0 \mathrm{eV}$.

unperturbed wavefunction of an electron delocalized over this bilayer.

$\hat{H}_{0}\left|\psi_{\vec{k} n}^{0}\right\rangle=E_{\vec{k} n}^{0}\left|\psi_{\vec{k} n}^{0}\right\rangle$

We now turn on SOC, such that $\hat{H}=\hat{H}_{0}+\hat{H}_{\text {soc. The new }}$ eigenstate representing the electron hopping between $\mathrm{HM}$ and FM is given by

$\left|\psi_{\vec{k} n}\right\rangle=\left|\psi_{\vec{k} n}^{0}\right\rangle+\sum_{\vec{q}, m} \frac{\left\langle\psi_{\vec{k}+\vec{q} m}^{0}\left|\hat{H}_{\mathrm{SOC}}\right| \psi_{\vec{k} n}^{0}\right\rangle}{E_{\vec{k} n}^{0}-E_{\vec{k}+\vec{q} m}^{0}}\left|\psi_{\vec{k}+\vec{q} m}^{0}\right\rangle$

Without loss of generality, we can assume the unperturbed FM has a magnetization along the $z$-axis and the perturbed magnetic moment rotates in the $\mathrm{XZ}$ plane. We now consider the component of magnetic moment of the perturbed state along $\vec{x}$, up to first order

$$
\left\langle\hat{s}_{x}\right\rangle=\sum_{\vec{q}, m}\left\langle\psi_{\vec{k} n}^{0}\left|\hat{S}_{x}\right| \psi_{\vec{k}+\vec{q} m}^{0}\right\rangle \frac{\left\langle\psi_{\vec{k}+\vec{q} m}^{0}\left|\hat{H}_{\mathrm{SOC}}\right| \psi_{\vec{k} n}^{0}\right\rangle}{E_{\vec{k} n}^{0}-E_{\vec{k}+\vec{q} m}^{0}}+C . C .
$$

Taking the long wavelength limit of $\vec{q} \rightarrow 0$.

$$
\left\langle\hat{s}_{x}\right\rangle=\sum_{m \neq n}\left\langle\psi_{\vec{k} n}^{0}\left|\hat{s}_{x}\right| \psi_{\vec{k} m}^{0}\right\rangle \frac{\left\langle\psi_{\vec{k} m}^{0}\left|\hat{H}_{\mathrm{SOC}}\right| \psi_{\vec{k} n}^{0}\right\rangle}{E_{\vec{k} n}^{0}-E_{\vec{k} m}^{0}}+C . C .
$$

Writing $\hat{s}_{x}$ and $\hat{H}_{\text {SOC }}$ in the spin basis $(|\uparrow\rangle,|\downarrow\rangle)$ :

$$
|\uparrow\rangle=\left[\begin{array}{l}
1 \\
0
\end{array}\right]|\downarrow\rangle=\left[\begin{array}{l}
0 \\
1
\end{array}\right] \hat{s}_{x}=\left[\begin{array}{ll}
0 & 1 \\
1 & 0
\end{array}\right] \hat{H}_{\mathrm{SOC}}=\left[\begin{array}{cc}
\lambda \hat{I}_{0} / 2 & \lambda \hat{I}_{-} / 2 \\
\lambda \hat{I}_{+} / 2 & -\lambda \hat{I}_{0} / 2
\end{array}\right]
$$




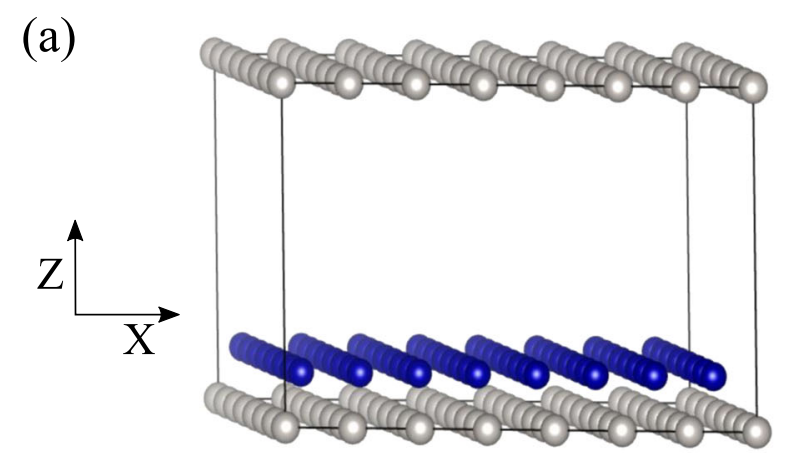

(b)

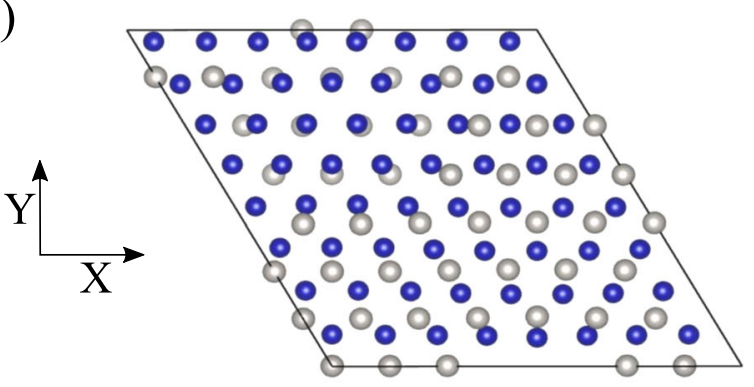

(c)

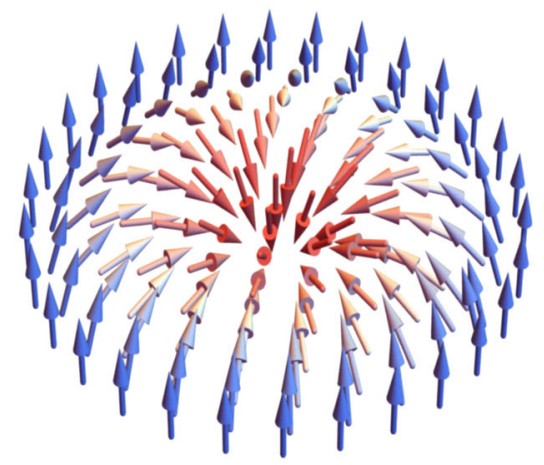

Fig. 4 Schematics of crystal structure and a skyrmion. Panels $\mathbf{a}$ and $\mathbf{b}$ demonstrate a schematic of our structure of $\mathrm{Pt} / \mathrm{Co}$ bilayer, where a shows the front view of the prerelaxed structure, which includes $10 \AA$ of vacuum, and $\mathbf{b}$ shows the top view of the postrelaxed structure. Panel c shows a schematic of a Neel skyrmion.

where $\hat{l}_{ \pm} \equiv \hat{l}_{x} \pm \hat{l}_{y} \quad \hat{l}_{0} \equiv \hat{l}_{z}$. We can write $\hat{l}_{ \pm}$in the d-orbital basis $\left(d_{z^{2}}, d_{x z}, d_{y z}, d_{x y}, d_{x^{2}-y^{2}}\right)$ as,

$\hat{l}_{+}=\left[\begin{array}{ccccc}0 & \sqrt{3} & 1 \sqrt{3} & 0 & 0 \\ -\sqrt{3} & 0 & 0 & l & 1 \\ -l \sqrt{3} & 0 & 0 & 1 & -l \\ 0 & -l & -1 & 0 & 0 \\ 0 & -1 & 1 & 0 & 0\end{array}\right] \hat{l}_{-}=\left[\begin{array}{ccccc}0 & -\sqrt{3} & 1 \sqrt{3} & 0 & 0 \\ \sqrt{3} & 0 & 0 & l & -1 \\ -l \sqrt{3} & 0 & 0 & -1 & -l \\ 0 & -l & 1 & 0 & 0 \\ 0 & 1 & 1 & 0 & 0\end{array}\right]$

For an unperturbed wavefunction $\psi_{\vec{k} n}^{0}$ in the spin-up state, the only nonzero contributions to $\left\langle\hat{s}_{x}\right\rangle$ come from $\psi_{\vec{k} m}^{0}$ in the spindown state.

$$
\begin{aligned}
\left|\psi_{\vec{k} n}^{0}\right\rangle & =\left|\phi_{\vec{k} n}^{0}, \uparrow\right\rangle ;\left|\psi_{\vec{k} m}^{0}\right\rangle=\left|\phi_{\vec{k} m}^{0}, \downarrow\right\rangle \\
\left\langle\hat{s}_{x}\right\rangle & =\lambda / 2 \sum_{m \neq n} \frac{\left\langle\phi_{\vec{k} m}^{0}\left|\hat{I}_{+}\right| \phi_{\vec{k} n}^{0}\right\rangle}{E_{\vec{k} n}^{0}-E_{\vec{k} m}^{0}}+C . C .
\end{aligned}
$$

where $\phi_{\vec{k}}^{0}$ is the orbital part of the wavefunction. Similarly, for an unperturbed wavefunction $\psi_{\vec{k} n}^{0}$ in the spin-down state, we get:

$$
\begin{aligned}
\left|\psi_{\vec{k} n}^{0}\right\rangle & =\left|\phi_{\vec{k} n}^{0}, \downarrow\right\rangle ;\left|\psi_{\vec{k} m}^{0}\right\rangle=\left|\phi_{\vec{k} m}^{0}, \uparrow\right\rangle \\
\left\langle\hat{S}_{x}\right\rangle & =\lambda / 2 \sum_{m \neq n} \frac{\left\langle\phi_{\vec{k} m}^{0}\left|\hat{I}_{-}\right| \phi_{\vec{k}}^{0}\right\rangle}{E_{\vec{k} n}^{0}-E_{\vec{k} m}^{0}}+C . C .
\end{aligned}
$$

Rotation of the magnetic moment of an electron, delocalized over the HM/FM interface, is caused by the spin orbit terms, $\hat{l}_{+} \cdot \hat{s}_{-}$and $\hat{I}_{-} \cdot \hat{s}_{+}$. To obtain a continuous, self-sustained rotation in real space, spin-up and spin-down states should be rotated such that they pick up magnetic moments in opposite directions along the $x$-axis. This condition requires $\left\langle\phi_{\vec{k} m}^{0}\left|\hat{I}_{+}\right| \phi_{\vec{k} n}^{0}\right\rangle$ to be opposite in sign to $\left\langle\phi_{\vec{k} m}^{0}\left|\hat{I}_{-}\right| \phi_{\vec{k} n}^{0}\right\rangle$. Inspecting Eq. (6), we conclude that there are only three such terms, namely, $\left\langle d_{x z}\left|\hat{I}_{ \pm}\right| d_{z^{2}}\right\rangle,\left\langle d_{x y}\left|\hat{I}_{ \pm}\right| d_{y z}\right\rangle$ and $\left\langle d_{x 2-y 2}\left|\hat{l}_{ \pm}\right| d_{x z}\right\rangle$.

To further illustrate this mechanism, we consider a wavefunction hopping across the HM/FM interface. In the presence of SOC, whenever the electron hops to the HM atom, the three SOC spin mixing terms cause d-orbital transitions and rotate its magnetic moment (creating a swirling spin texture). As this electron hops to the FM, its rotated magnetic moment perturbs the wavefunctions of the FM via exchange interaction, altering the free energy and generating $\mathrm{DMI}^{40}$. The stronger the HM-FM hybridization, the stronger the perturbation of energy due to the hopping electron, the larger the DMI. The sign of DMI is decided by the interplay between these three SOC spin mixing terms and the strength of DMI is decided by the HM-FM hybridization. We note that this result does not support the model of ${ }^{10}$ where the source of DMI is identified to be transitions between $d_{x z}$ and $d_{y z}$ orbitals.

We now employ our theory to explain the trends of DMI sign observed in our calculations, thereby shining light on previously unexplained materials behaviour. According to our theory, three SOC transition terms, generate three separate DMI terms. The direction of each SOC transition will decide the sign of the corresponding DMI term, with opposite directions of transition leading to opposite DMI signs. The direction and strength of a transition, in turn, depends on the relative presence of HM orbitals $\left(d_{1}\right.$ and $\left.d_{2}\right)$ in the bandstructure, before SOC is turned on. Specifically, the stronger the presence of $d_{1}$, and the weaker the presence of $d_{2}$, the stronger the transition from $d_{1}$ to $d_{2}$ would be, once SOC is turned on. The result of these inferences is that if we compare materials with similar crystal structures, their bandstructures and energy denominators $\left(E_{\vec{k} n}^{0}-E_{\overrightarrow{k m}}^{0}\right)$ are similar too. Consequently, it is the variation of relative presence of $\mathrm{HM} \mathrm{d}$ orbitals that controls the net DMI sign.

With this in mind, we compare the contribution of $d$ orbitals to the bandstructure for some chosen bilayers (Re/Fe, Os/Fe, Ir/Fe, $\mathrm{Re} / \mathrm{Co}, \mathrm{Os} / \mathrm{Co}, \mathrm{Pt} / \mathrm{Co}, \mathrm{Os} / \mathrm{Ni}$ and $\mathrm{Pt} / \mathrm{Ni}$ ). These bilayers were selected from the bigger dataset for their large values of DMI and similar crystal structures. This enabled us to compare them to one another, as well as, easily demonstrate the mechanism behind DMI. We visually inspected the projected bandstructures in these materials, and found that the SOC transitions occur close to the $\mathrm{K}$ point in the Brillouin zone (BZ). The specific energy ranges over which the transitions occurred varied for different materials, but were generally found to be between -3 to $-1 \mathrm{eV}$, or -4 to $-2 \mathrm{eV}$ below the Fermi level. Figure. 5 plots the difference in orbital contributions ( $p$-DOS) from HM d orbitals around the $\mathrm{K}$ point, in the energy range corresponding to the bilayers. Keeping in mind that orbital contributions can only roughly capture SOC transitions, they turn out to be a good qualitative predictor of the sign of DMI. They also help explain why the three outliers mentioned above have DMI smaller than that predicted by $\mathrm{BO}$ alone.

With regards to Fig. 5 , a positive value of $d_{1}-d_{2}$ contribution, signifies a likely transition from $d_{1}$ to $d_{2}$ and vice versa. For our 

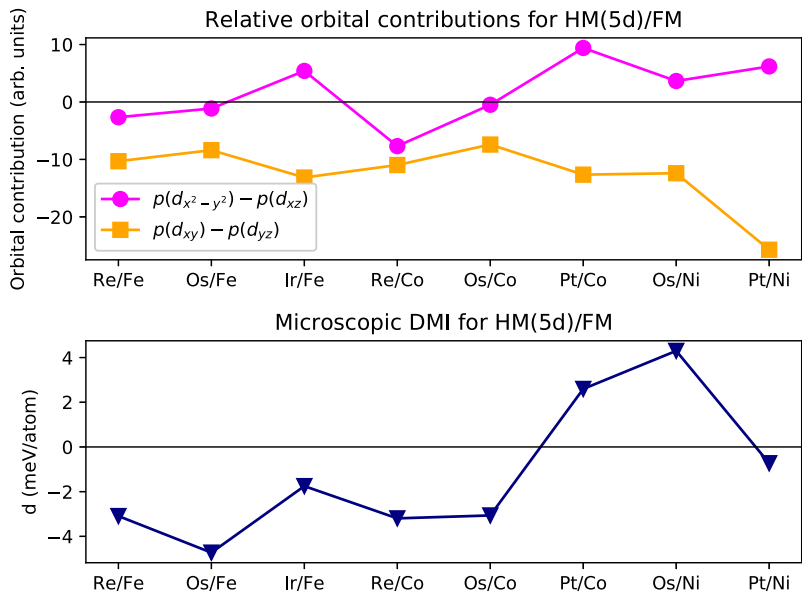

Fig. 5 Plots for difference in orbital contributions (around $\mathrm{K}$ ), and microscopic DMI. The difference between contributions of $d_{x^{2}-y^{2}}$ and $d_{x z}$ is plotted with magenta circles, and that between contributions of $d_{x y}$ and $d_{y z}$ is plotted with orange squares. Microscopic DMl is plotted using navy blue triangles for comparision.

chosen bilayers, we infer that $d_{y z} \rightarrow d_{x y}$ and $d_{x z} \rightarrow d_{x^{2}-y^{2}}$ transitions will generate negative DMI terms leading to counterclockwise rotation of spin. We observe from Fig. 5 that all our chosen materials show a $d_{y z} \rightarrow d_{x y}$ transition and a corresponding negative DMI term. However, early HMs tend to show a $d_{x z} \rightarrow$ $d_{x^{2}-y^{2}}$ transition (negative DMl term) whereas late HMs show the reverse $d_{x^{2}-y^{2}} \rightarrow d_{x z}$ transition (positive DMI term). The sum of these DMI terms gives the final DMI. In Re/Fe, Os/Fe and Re/Co, both DMI terms are negative leading to a large negative net DMI. However, in $\mathrm{Ir} / \mathrm{Fe}, \mathrm{Pt} / \mathrm{Co}$ and $\mathrm{Pt} / \mathrm{Ni}$, the two DMl terms have opposite sign and compete with one another. This explains why these outliers have a lower DMI than expected simply from $\mathrm{BO}$. We do not observe any $\left\langle d_{x z}\left|\hat{I}_{ \pm}\right| d_{z^{2}}\right\rangle$ transitions. We note that Os/Ni also has competing transitions but shows a larger than expected DMI. Despite this exception, overall, the relative $d$-orbital contributions are a good qualitative predictor of the sign of DMI.

Our work has important implications as to the understanding of DMI and the control of skyrmions. First, our model also explains the correlation between DMI and orbital anisotropy reported recently ${ }^{43}$. According to our theory, DMl originates in spin mixing orbital transitions caused by SOC. Such transitions along with generating DMI will also naturally distort orbital shapes and alter orbital moments, leading to the correlation between DMI and orbital asymmetry observed. Second, our model suggests that DMI in bilayers can be controlled by tuning the HM-FM hybridization. A similar concept has been proposed for bulk $\epsilon \mathrm{Co}^{44}$. Third, the engineering of relative $\mathrm{HM}-5 \mathrm{~d}$ orbital fillings, via strain and symmetry breaking, could provide a pathway for controlling the sign of DMI and the chirality of skyrmions. Control of skyrmions with an electric field would be a tremendous leap forward in the development of skyrmion technology.

\section{METHODS}

Density functional theory calculations

We calculated the DMI using density functional theory (DFT) for a comprehensive series of HM/FM bilayers. Our choice of HM varied through the $5 \mathrm{~d}$ and $6 \mathrm{p}$ series of elements, ranging from $\mathrm{Hf}$ to $\mathrm{Bi}$. We chose the $\mathrm{FM}$ to be $\gamma$ - $\mathrm{Fe}(111), \mathrm{Co}(0001)$ or $\mathrm{Ni}(111)$. DFT calculations were performed using Vienna ab initio Simulation Package (VASP) ${ }^{45-47}$ with PAW-PBE pseudopotentials ${ }^{48}$. Taking the initial bulk structures from literature, we optimized their volume and constructed bilayers comprising of a monolayer of FM, a monolayer of $\mathrm{HM}$ and $10 \AA$ of vacuum. A schematic of these structures is shown in Fig. 4. As the DMI in these bilayers is known to be interfacial ${ }^{11}$, using monolayers of FM and HM is sufficient to capture the essential physics. The in-plane lattice constant was fixed to that of the $\mathrm{HM}$ and the FM was strained to less than $5 \%$ to match. The calculations were converged with respect to kmesh and plane wave energy cut-offs. All structures were then relaxed till the Hellman-Feynman forces on all atoms were less than $0.01 \mathrm{eV} / \AA$. . DMI was the calculated using the mechanism described by Yang et al. ${ }^{8}$. The microscopic DMI (d) obtained from first principles was used to further calculate the micromagnetic DMI $(D)$. An important caveat is that our calculations for DMl are performed with the assumption that the nearest neighbour (NN) DMI term is the dominant contribution to the total DMI. We make this assumption based on the expectation that the hopping terms between NN FM atoms (via HM), will in general be stronger than the hopping terms between next-nearest neighbours (NNN) FM atoms (via HM). According to our model, larger hopping terms will generally lead to stronger DMI. This picture should hold for our structures where there is a significant lattice mismatch between HM and FM layers and strong structural relaxation. It has been shown for chiral bulk materials ${ }^{49}$, that NNN DMI terms become less important than dominant NN DMI terms at nonzero magnetic fields. Therefore, we expect our results to be relevant to obtaining room temperature, small sized skyrmions at nonzero magnetic fields.

\section{DATA AVAILABILITY}

The data that support the findings of this study are available upon request to the corresponding author.

\section{CODE AVAILABILITY}

The central code used in this study is Vienna Ab initio Simulation Package (VASP). Further information regarding licensing and code documentation can be found at https://cms.mpi.univie.ac.at/wiki/index.php.

Received: 28 May 2019; Accepted: 20 May 2020;

Published online: 03 July 2020

\section{REFERENCES}

1. Moreau-luchaire, C. Additive interfacial chiral interaction in multilayers for stabilization of small individual skyrmions at room temperature. Nat. Nanotechnol. 11, 444-448 (2016).

2. Fert, A., Reyren, N. \& Cros, V. Magnetic skyrmions: advances in physics and potential applications. Nat. Rev. Mater. 2, 17031 (2017).

3. Fert, A., Cros, V. \& Sampaio, J. Skyrmions on the track. Nat. Nanotechnol. 8, 152 (2013).

4. Bode, M. Chiral magnetic order at surfaces driven by inversion asymmetry. Nature 447, 190 (2007).

5. Dzyaloshinsky, I. A thermodynamic theory of weak ferromagnetism of antiferromagnetics. J. Phys. Chem. Solids 4, 241-255 (1958).

6. Moriya, T. Anisotropic superexchange interaction and weak ferromagnetism Phys. Rev. 120, 91-98 (1960).

7. Ryu, K.-S., Yang, S.-H., Thomas, L. \& Parkin, S. S. P. Chiral spin torque arising from proximity-induced magnetization. Nat. Commun. 5, 3910 (2014).

8. Yang, H., Thiaville, A., Rohart, S., Fert, A. \& Chshiev, M. Anatomy of DzyaloshinskiiMoriya interaction at Co/Pt interfaces. Phys. Rev. Lett. 115, 267210 (2015).

9. Belabbes, A., Bihlmayer, G., Bechstedt, F., Blügel, S. \& Manchon, A. Hund's ruledriven Dzyaloshinskii-Moriya interaction at 3d-5d interfaces. Phys. Rev. Lett. 117, 247202 (2016).

10. Kashid, V., Shah, V. \& Salunke, H. G. Dzyaloshinskii-Moriya interaction and chiral magnetism in $3 d-5 d$ zigzag chains: Tight-binding model and ab initio calculations. Phys. Rev. B 90, 054412 (2014).

11. Yang, H., Boulle, O., Cros, V., Fert, A. \& Chshiev, M. Insulator capping and electric field. Sci. Rep. 8, 12356 (2018)

12. Heinze, S. Spontaneous atomic-scale magnetic skyrmion lattice in two dimensions. Nat. Phys. 7, 713 (2011)

13. Tolley, R., Montoya, S. A. \& Fullerton, E. E. Room-temperature observation and current control of skyrmions in Pt/Co/Os/Pt thin films. Phys. Rev. Mater 2, 044404 (2018).

14. Belmeguenai, M. Interfacial Dzyaloshinskii-Moriya interaction in perpendicularly magnetized $P t / C o / A / O_{x}$ ultrathin films measured by Brillouin light spectroscopy. Phys. Rev. B 91, 180405 (2015).

15. Chen, G. Tailoring the chirality of magnetic domain walls by interface engineering. Nat. Commun. 4, 2671 (2013) 
16. Jiang, W., Chen, G., Liu, K., Zang, J., te Velthuise, S. G. E. \& Hoffmann, A. Skyrmions in magnetic multilayers. Phys. Rep. 704, 1-49 (2017).

17. Romming, N. Writing and deleting single magnetic skyrmions. Science $\mathbf{3 4 1}$, 636-639 (2013).

18. Meyer, S. Isolated zero field sub-10?nm skyrmions in ultrathin Co films. Nat. Commun. 10, 3823 (2019).

19. Soumyanarayanan, A. Tunable room-temperature magnetic skyrmions in $\mathrm{Ir} / \mathrm{Fe} /$ Co/Pt multilayers. Nat. Mater. 16, 898 (2017).

20. Boulle, O. Room-temperature chiral magnetic skyrmions in ultrathin magnetic nanostructures. Nat. Nanotechnol. 11, 449-454 (2016).

21. Ho, P. Geometrically tailored skyrmions at zero magnetic field in multilayered nanostructures. Phys. Rev. Appl. 11, 024064 (2019).

22. Woo, S. Observation of room temperature magnetic skyrmions and their current driven dynamics in ultrathin metallic ferromagnets. Nat. Mater. 15, 501 (2016).

23. Okubo, T., Chung, S. \& Kawamura, H. Multiple-q states and the skyrmion lattice of the triangular-lattice Heisenberg antiferromagnet under magnetic fields. Phys. Rev. Lett. 108, 017206 (2012).

24. Leonov, A. O. \& Mostovoy, M. Multiply periodic states and isolated skyrmions in an anisotropic frustrated magnet. Nat. Comm. 6, 8275 (2015).

25. Hayami, S., Lin, S.-Z. \& Batista, C. D. Bubble and skyrmion crystals in frustrated magnets with easy-axis anisotropy. Phys. Rev. B 93, 184413 (2016).

26. Liang, J. J. Magnetic field gradient driven dynamics of isolated skyrmions and antiskyrmions in frustrated magnets. N. J. Phys. 20, 053037 (2018).

27. Kurumaji, T. Skyrmion lattice with a giant topological Hall effect in a frustrated triangular-lattice magnet. Science 10, 1126 (2019).

28. Bogdanov, A. \& Hubert, A. Thermodynamically stable magnetic vortex states in magnetic crystals. J. Magn. Magn. Mater. 138, 255 (1994).

29. Ezawa, M. Giant skyrmions stabilized by dipole-dipole interactions in thin ferromagnetic films. Phys. Rev. Lett. 105, 197202 (2010).

30. Rohart, S. \& Thiaville, A. Skyrmion confinement in ultrathin film nanostructures in the presence of Dzyaloshinskii-Moriya interaction. Phys. Rev. B 88, 184422 (2013).

31. Romming, N., Kubetzka, A., Hanneken, C. \& Wiesendanger, R. Field-dependent size and shape of single magnetic skyrmions. Phys. Rev. Lett. 114, 177203 (2015).

32. Dupé, B., Bihlmayer, G., Böttcher, M., Blügel, S. \& Heinze, S. Engineering skyrmions in transition-metal multilayers for spintronics. Nat. Comm. 7, 11779 (2016).

33. Cho, J. Thickness dependence of the interfacial Dzyaloshinskii-Moriya interaction in inversion symmetry broken systems. Nat. Comm. 6, 7635 (2015).

34. Soumyanarayanan, A., Reyren, N., Fert, A. \& Panagopoulos, C. Emergent phenomena induced by spin orbit coupling at surfaces and interfaces. Nature 539, 509 (2016).

35. Simon, E. Spin-correlations and magnetic structure in an Fe monolayer on 5d transition metal surfaces. J. Phys. Condens. Matter 26, 186001 (2014).

36. Kloodt-Twesten, F., Kuhrau, S. \& Frömter, R. Measuring the Dzyaloshinskii-Moriya interaction of the epitaxial Co/lr(111) interface. Phys. Rev. B 100, 100402 (2019).

37. Reis, F. Bismuthene on a SiC substrate: a candidate for a high-temperature quantum spin Hall material. Science 357, 287-290 (2017).

38. Dupé, B., Bihlmayer, G., Böttcher, M., Blügel, S. \& Heinze, S. Engineering skyrmions in transition metal multilayers for spintronics. Nat. Commun. 7, 11779 (2016).

39. Hrabec, A. Measuring and tailoring the Dzyaloshinskii-Moriya interaction in perpendicularly magnetized thin films. Phys. Rev. B 90, 020402 (2014).

40. Freimuth, F., Blügel, S. \& Mokrousov, Y. Berry phase theory of Dzyaloshinskii-Moriya interaction and spin-orbit torques. J. Phys. Condens. Matter 26, 104202 (2014).

41. Hanke, J.-P., Freimuth, F., Blügel, S. \& Mokrousov, Y. Higher-dimensional Wannier interpolation for the modern theory of the Dzyaloshinskii-Moriya interaction: application to Co-based trilayers. J. Phys. Soc. Jpn. 87, 041010 (2018).

42. Hoffmann, A. \& Bader, S. D. Opportunities at the frontiers of spintronics. Phys. Rev. Appl. 4, 047001 (2015).

43. Kim, S. Correlation of the Dzyaloshinskii-Moriya interaction with Heisenberg exchange and orbital asphericity. Nat. Comm. 9, 1648 (2018).
44. Luo, H.-B., Zhang, H.-B. \& Liu, J. P. Strong hopping induced Dzyaloshinskii-Moriya interaction and skyrmions in elemental cobalt. npj Comput. Mater. 5, 50 (2019).

45. Kresse, G. \& Hafner, J. Ab initio molecular dynamics for liquid metals. Phys. Rev. $B$ 47, 558 (1993).

46. Kresse, G. \& Furthmüller, J. Efficiency of ab-initio total energy calculations for metals and semiconductors using a plane-wave basis set. Comput. Mater. Sci. 6, 15-50 (1996).

47. Kresse, G. \& Furthmüller, J. Efficient iterative schemes for ab initio total-energy calculations using a plane-wave basis set. Phys. Rev. B 54, 11169 (1996).

48. Kresse, G. \& Joubert, D. From ultrasoft pseudopotentials to the projector augmented-wave method. Phys. Rev. B 59, 1758 (1999).

49. Oliveira, E. A. S., Silva, R. L., Silva, R. C. \& Pereira, A. R. Effects of second neighbor interactions on skyrmion lattices in chiral magnets. J. Phys. Condens. Matter 29, 205801 (2017).

\section{ACKNOWLEDGEMENTS}

We would like to thank Qian Niu for useful discussions on the microscopic model for DMI and NSF for financial support under the grants NNCI ECCS-1542159, EFRInewLAW and NASCENT ERC. We also acknowledge the Texas Advanced Computing Center (TACC) at The University of Texas at Austin for providing HPC resources that have contributed to the research results reported within this paper (http://www.tacc. utexas.edu).

\section{AUTHOR CONTRIBUTIONS}

P.J. designed the project, performed the calculations, derived the theory and wrote the initial manuscript. All authors discussed the results and the derivation, as well as contributed to the final version of manuscript.

\section{COMPETING INTERESTS}

The authors declare no competing interests.

\section{ADDITIONAL INFORMATION}

Correspondence and requests for materials should be addressed to P.J.

Reprints and permission information is available at http://www.nature.com/ reprints

Publisher's note Springer Nature remains neutral with regard to jurisdictional claims in published maps and institutional affiliations.

\begin{abstract}
(c) (1)
Open Access This article is licensed under a Creative Commons Attribution 4.0 International License, which permits use, sharing, adaptation, distribution and reproduction in any medium or format, as long as you give appropriate credit to the original author(s) and the source, provide a link to the Creative Commons license, and indicate if changes were made. The images or other third party material in this article are included in the article's Creative Commons license, unless indicated otherwise in a credit line to the material. If material is not included in the article's Creative Commons license and your intended use is not permitted by statutory regulation or exceeds the permitted use, you will need to obtain permission directly from the copyright holder. To view a copy of this license, visit http://creativecommons. org/licenses/by/4.0/.
\end{abstract}

(c) The Author(s) 2020 